Proceedings the 44th International School and Conference on the Physics of Semiconductors "Jaszowiec 2015", Wisła 2015

\title{
Type-I and Type-II Confinement in Quantum Dots: Excitonic Fine Structure
}

\author{
V. KŘÁPEK ${ }^{a, *}$, P. KLENOVSKÝ ${ }^{b}$, T. ŠIKOLA ${ }^{a, c}$ \\ ${ }^{a}$ Central European Institute of Technology, Brno University of Technology, \\ Technická 10, 61600 Brno, Czech Republic \\ ${ }^{b}$ Central European Institute of Technology, Masaryk University, Kamenice 753, 62500 Brno, Czech Republic \\ ${ }^{c}$ Institute of Physical Engineering, Brno University of Technology, Technická 2, 61669 Brno, Czech Republic \\ We have theoretically studied type-I and type-II confinement in InAs quantum dots with $\mathrm{GaAs}_{1-y} \mathrm{Sb}_{y}$ capping \\ layer. The character of the confinement can be adjusted by the Sb content. We have found that upon the transition \\ from type-I to type-II confinement the hole wave functions change the topology from a compact shape to a two- \\ segment shape, resulting in the complex changes in the exciton fine structure splitting with zero values at particular \\ compositions. Additionally, a high exciton radiative recombination probability is preserved even in type-II. This \\ allows to design strongly luminescent quantum dots with naturally low fine structure splitting, which could serve \\ as sources of entangled photon pairs for quantum communication.
}

DOI: 10.12693/APhysPolA.129.A-66

PACS: 71.35.--y, 73.21.La, 81.05.Ea

\section{Introduction}

The ability of the quantum dots to isolate and manipulate individual charge carriers and their integrability into more complex devices make them a candidate for various applications in field of quantum information processing. The source of entangled photon pairs, a crucial element of distinct quantum communication protocols, has been proposed [1] and later realized [2] using the biexciton-exciton radiative cascade. For this it is important that the splitting of the bright exciton doublet (so called fine structure splitting, FSS) is lower than the transition linewidth. FSS was attributed to the anisotropic electron-hole exchange interaction [3] which emerges when the symmetry is lower than $D_{2 d}[4]$. FSS of as-grown structures is usually by far too large and elaborate methods are employed in order to reduce it. The usual lateral asymmetry is suppressed by the growth of symmetric strain-free quantum dots [5] or by the growth on [111] substrates [6]. Post-growth tuning of FSS is possible by means of the external electric [7], magnetic [4], or strain field $[8,9]$.

Type-II QDs confine one type of charge carriers in the QD volume while the other type resides in a barrier and is only loosely bound by local variations of the confinement potential or the Coulomb coupling to the carrier inside the QD. Here we focus on InAs QDs with $\mathrm{GaAs}_{1-y} \mathrm{Sb}_{y}$ capping layer (CL), in which holes are localized in the barrier close to the vicinity of the QD. The type of confinement can be set by the $\mathrm{Sb}$ content $y$; the transition

\footnotetext{
* corresponding author; e-mail: vlastimil.krapek@ceitec.vutbr.cz
}

values between 0.14 and 0.18 have been reported [10, 11]. The photoluminescence of the QDs is rather strong even in type-II regime up to $y=0.28$ [12].

In this work we study theoretically the properties of InAs QDs with the $\mathrm{GaAs}_{1-y} \mathrm{Sb}_{y}$ CL in type-I and typeII confinement regimes. We have demonstrated that with increasing $\mathrm{Sb}$ content the smooth crossover between typeI and type-II regimes is induced, accompanied by the red shift of the transition energy, the segmentation of the hole wave function, and non-trivial changes in the FSS magnitude and polarization of the bright exciton transitions. We predict the possibility to prepare QDs with naturally low FSS and efficient photoluminescence by setting a proper Sb content.

\section{Theory}

The single particle states were calculated within the eight-band $\boldsymbol{k} \cdot \boldsymbol{p}$ theory [13] using the Nextnano 3D software package [14]. Realistic strain and piezoelectric fields were included. Next, the exciton states were calculated using the configuration interaction method [15] in which the wave functions are expanded into a basis of Slater determinants formed from the single particle states. The matrix elements of the Coulomb Hamiltonian were calculated following the approach of Refs. [3, 16].

\section{Results}

We consider here a lens-shaped InAs QD with the height of $4 \mathrm{~nm}$ and the radius of $8 \mathrm{~nm}$ on GaAs substrate, capped with a $\mathrm{GaAs}_{1-y} \mathrm{Sb}_{y}$ layer with the thickness of $6 \mathrm{~nm}$. The axis of the lens is parallel with the [001] crystal axis, the related coordinate is denoted $z$. To induce the crossover between the type-I and type-II confinement regimes, we have varied the antimony content $y$ between 
0.1 and 0.22 . The bulk valence band edges of InAs and $\mathrm{GaAs}_{1-y} \mathrm{Sb}_{y}$ cross for $y_{\mathrm{CO}} \approx 0.15$ with the InAs valence band being higher in energy (i.e., confining the holes) for $y<y_{\mathrm{CO}}$.
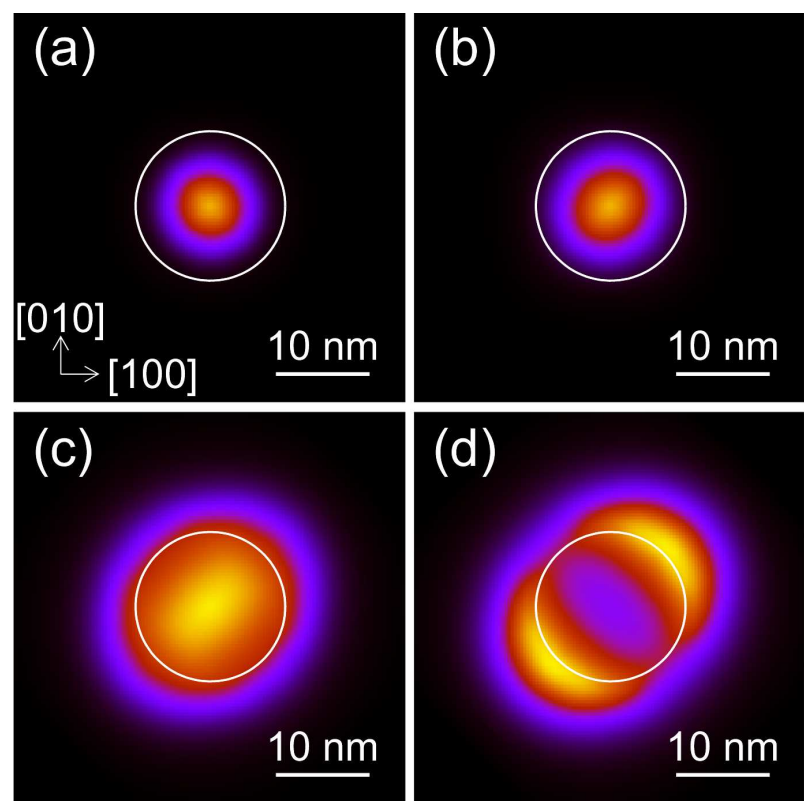

Fig. 1. In-plane probability density (integrated over $z$ ) of the ground state of (a) electron, $y=0.10$, (b) hole, $y=0.10$, (c) hole, $y=0.20$, (d) hole, $y=0.22$. White circles denote the QD boundary at the height of the base.

We will first inspect the wave functions. In-plane probability densities (quadrate moduli of the wave functions integrated over direction $z$ parallel with the lens axis) are shown in Fig. 1. The electrons (Fig. 1a) are localized inside the QD volume, slightly elongated along [1 10$]$ due to the piezoelectric field, and remain practically invariant under the change of $\mathrm{Sb}$ content $y$. Holes are for lower antimony content also localized within the QD volume (Fig. 1b) and slightly elongated along [110]. As $y$ increases, the hole wave function extends laterally more into barrier (Fig. 1c). Finally, holes are expelled from the $\mathrm{QD}$ volume and become confined in the $\mathrm{GaAs}_{1-y} \mathrm{Sb}_{y}$ layer (Fig. 1d), where their wave function forms two segments [11] located along the [110] direction, each elongated along [110]. The vertical position and extension of the holes undergo negligible variations during the transition between type-I and type-II regimes.

To describe the type-I-type-II crossover quantitatively, we introduce the probability of finding a particle inside the QD volume (i.e., within InAs). It is shown in Fig. 2a for electrons and holes. While the electron probability displays only negligible variations of less than $1 \%$, the hole probability gradually decreases from $85 \%$ at $y=0.10$ to $19 \%$ at $y=0.22$, reaching $50 \%$ for $y=0.17$ which can be thus considered as the crossover value of the $\mathrm{Sb}$ content for the particular nanostructure. Nevertheless, the transition between type-I and type-II regimes

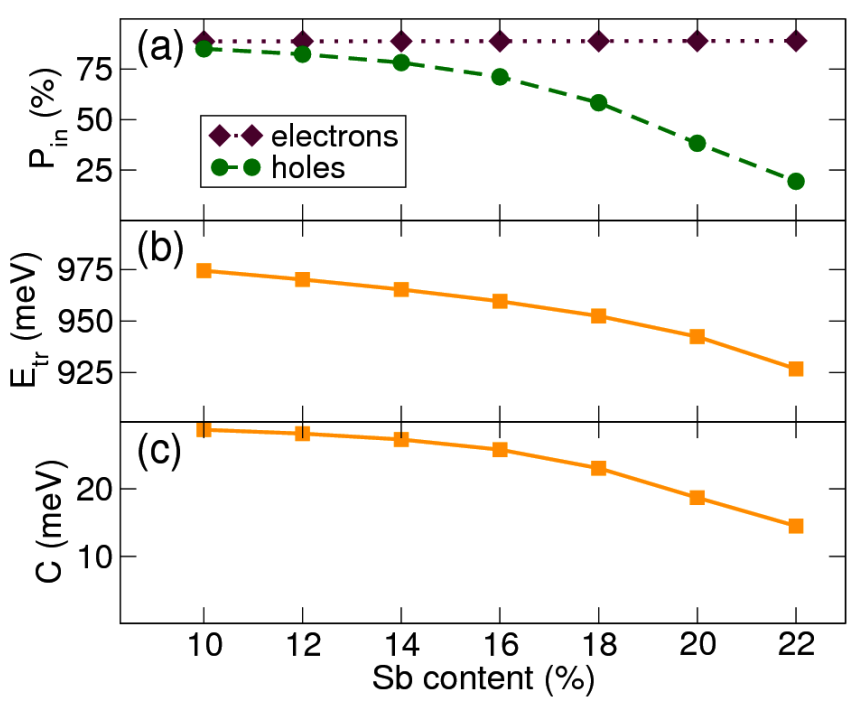

Fig. 2. (a) Probability of finding a particle inside the QD volume, (b) transition energy of the ground exciton state, (c) Coulomb energy between the electron and hole ground states.

is smooth and no abrupt changes are observed. The transition energy redshifts as the $y$ increases (Fig. 2b) due to the decreased potential barrier in type-I and the shift of the confinement potential edge in type-II and some other effects. The red shift has been already observed [17] and theoretically explained [11]. An important consequence of the electron-hole separation in type-II is the reduced electron-hole Coulomb coupling. The direct coupling energy, which is equal to the binding energy of exciton and contributes the binding energy of excitonic complexes, is shown in Fig. 2c; it gradually decreases from 29 to $15 \mathrm{meV}$. The decrease is first subtle, caused by the increased extension of the hole wave function that is still localized inside the QD, and becomes steeper when charge carriers are separated and two-segmented hole wave function outside the QD is formed. A dependence of FSS on Sb content is shown in Fig. 3. The FSS evolution is dominated by the variations of the lateral symmetry of the wave functions and further contributed by a reduction of the exchange Coulomb coupling due to the separation of electron and hole wave function with increasing $y$ (the latter is responsible for a kink at $y=0.18$ ). The polarization properties of the exciton transitions are encoded into the sign of FSS: positive (negative) sign corresponds to the linear polarization of the lower-energy exciton transition along [110] $([1 \overline{1} 0])$, respectively. Due to the symmetry of the crystal lattice and the structure, only these two polarizations are observed. The higherenergy transition is linearly polarized in perpendicular direction to the lower-energy transition. In terms of the wave function properties the polarization direction of the lower-energy transition corresponds to the long axis of the elongated wave function. In symmetric lens-shaped QDs, holes and electrons are elongated in perpendicular 


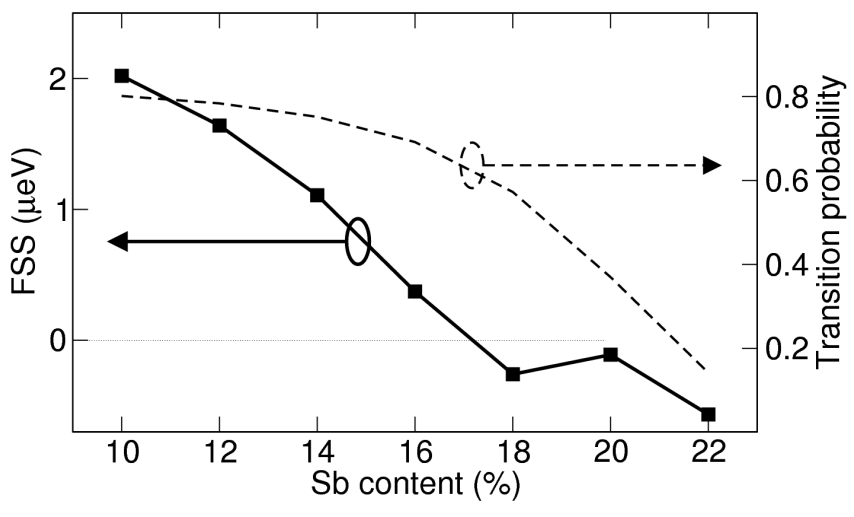

Fig. 3. FSS (thick solid line with symbols, left) and transition probability (thin dashed line, right) between the ground electron and hole state in the lens-shaped $\mathrm{QD}$ as functions of the GaAsSb layer composition.

directions, partly compensating the overall anisotropy. For $y$ below 0.16 , hole elongation along [110] dominates, resulting in positive FSS. Above 0.18, electron elongation along [1ํㅣ becomes stronger, changing the sign of FSS. Finally, after the formation of the segmented hole function, the hole segments are also elongated along [110], increasing the magnitude of FSS. Noteworthy, FSS acquires a zero value at $y \approx 0.17$, which is an essential ingredient for the realization of the polarization-entangled photon pairs [1]. In addition, $\mathrm{GaAs}_{1-y} \mathrm{Sb}_{y}$ capped InAs QDs are good emitters even in type-II regime. The calculated values of transition probability for the ground exciton recombination, shown in Fig. 3, exhibit only a moderate decrease with increasing Sb content with a reduction towards $25 \%$ of the type-I value for $y=0.22$. The observations of a strong photoluminescence in typeII regime [12] corroborate our prediction.

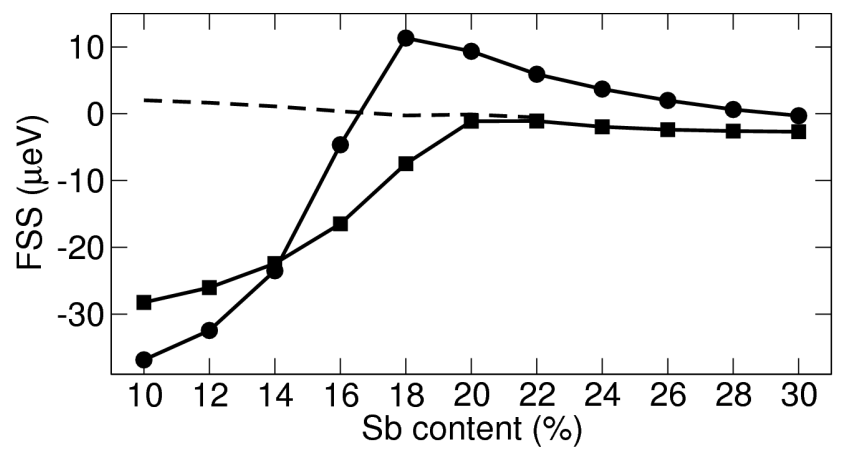

Fig. 4. FSS in the elongated lens-shaped QD as a function of the GaAsSb layer antimony content. The thickness of CL is $4 \mathrm{~nm}$ (squares) or $7 \mathrm{~nm}$ (circles). For comparison, FSS in symmetric lens-shaped QD with the CL height of $6 \mathrm{~nm}$ is shown (dashed line).

A reduction of FSS towards zero can be achieved also in more realistic non-symmetric QDs. In Fig. 4 we show FSS in lens-shaped QDs elongated along [1]0] with the ratio of the lateral dimensions of 1.1 for two thicknesses of the CL ( 4 and $7 \mathrm{~nm}$ ). The shape elongation causes the large negative FSS in type-I regime. Although FSS in type-I regime is large compared to a natural exciton linewidth (about $4 \mu \mathrm{eV}$ for a typical life time of $1 \mathrm{~ns}$ [12]), for both CL thicknesses it is reduced below the linewidth during the crossover to the type-II regime. Thus, by setting a proper composition of the $\mathrm{GaAs}_{1-y} \mathrm{Sb}_{y}$ overlayer, QDs with a low value of FSS can be produced.

\section{Conclusions}

The character of confinement in InAs quantum dots capped with a $\mathrm{GaAs}_{1-y} \mathrm{Sb}_{y}$ overlayer depends on the composition of the overlayer, with the holes coupled inside the QD for low Sb content and outside the QD for high $\mathrm{Sb}$ content. During the crossover between type-I and type-II confinement, the lateral profile of the hole wave functions is modified, resulting in the strong variations of the FSS. By setting a proper $\mathrm{Sb}$ content it shall be possible to prepare QDs with naturally low FSS and efficient photoluminescence, which are important for quantum communication as the source of polarizationentangled photon pairs.

\section{Acknowledgments}

This work was supported by European Social Fund (grant No. CZ.1.07/2.3.00/30.0005), European Regional Development Fund (project No. CZ.1.05/1.1.00/02.0068), the Grant Agency of the Czech Republic (grant No. 15-21581S), Technology Agency of the Czech Republic (grant No. TE01020233), and EU 7th Framework Programme (Contracts No. 286154 - SYLICA and 280566 - UnivSEM). P.K. was supported by the internal project MUNI/A/1496/2014.

\section{References}

[1] O. Benson, C. Santori, M. Pelton, Y. Yamamoto, Phys. Rev. Lett. 84, 2513 (2000).

[2] N. Akopian, N.H. Lindner, E. Poem, Y. Berlatzky, J. Avron, D. Gershoni, B.D. Gerardot, P.M. Petroff, Phys. Rev. Lett. 96, 130501 (2006).

[3] T. Takagahara, Phys. Rev. B 62, 16840 (2000).

[4] M. Bayer, G. Ortner, O. Stern, A. Kuther, A.A. Gorbunov, A. Forchel, P. Hawrylak, S. Fafard, K. Hinzer, T.L. Reinecke, S.N. Walck, J.P. Reithmaier, F. Klopf, F. Schäfer, Phys. Rev. B 65, 195315 (2002).

[5] Y.H. Huo, V. Křápek, A. Rastelli, O.G. Schmidt, Phys. Rev. B 90, 041304 (2014).

[6] A. Schliwa, M. Winkelnkemper, A. Lochmann, E. Stock, D. Bimberg, Phys. Rev. B 80, 161307 (2009).

[7] R. Trotta, E. Zallo, C. Ortix, P. Atkinson, J.D. Plumhof, J. van den Brink, A. Rastelli, O.G. Schmidt, Phys. Rev. Lett. 109, 147401 (2012).

[8] J.D. Plumhof, V. Křápek, F. Ding, K.D. Jöns, R. Hafenbrak, P. Klenovský, A. Herklotz, K. Dörr, P. Michler, A. Rastelli, O.G. Schmidt, Phys. Rev. B 83, 121302 (2011). 
[9] J.D. Plumhof, R. Trotta, V. Křápek, E. Zallo, P. Atkinson, S. Kumar, A. Rastelli, O.G. Schmidt, Phys. Rev. B 87, 075311 (2013).

[10] H.Y. Liu, M.J. Steer, T.J. Badcock, D.J. Mowbray, M.S. Skolnick, F. Suarez, J.S. Ng, M. Hopkinson, J.P.R. David, J. Appl. Phys. 99, 046104 (2006).

[11] P. Klenovský, V. Křápek, D. Munzar, J. Humlíček, Appl. Phys. Lett. 97, 203107 (2010).

[12] J.M. Ulloa, J.M. Llorens, B. Alén, D.F. Reyes, D.L. Sales, D. González, A. Hierro, Appl. Phys. Lett. 101, 253112 (2012).

[13] O. Stier, M. Grundmann, D. Bimberg, Phys. Rev. B 59, 5688 (1999).
[14] S. Birner, T. Zibold, T. Andlauer, T. Kubis, M. Sabathil, A. Trellakis, P. Vogl, IEEE Trans. Electron Dev. 54, 2137 (2007).

[15] R. Seguin, A. Schliwa, S. Rodt, K. Pötschke, U.W. Pohl, D. Bimberg, Phys. Rev. Lett. 95, 257402 (2005).

[16] V. Křápek, P. Klenovský, T. Šikola, Phys. Rev. B 92, 195430 (2015).

[17] A. Hospodková, M. Zíková, J. Pangrác, J. Oswald, J. Kubištová, K. Kuldová, P. Hazdra, E. Hulicius, J. Phys. D Appl. Phys. 46, 095103 (2013). 